\title{
Super-Kamiokande latest atmospheric neutrino results
}

\author{
Flor de María Blaszczyk* \\ Boston University \\ E-mail: fblaszcz@bu.edu
}

The latest results of Super Kamiokande's atmospheric neutrino analyses are presented. The first part focuses on the three-flavor neutrino oscillation analysis with and without external constraints, optimized for sensitivity to the neutrino mass hierarchy, including data from all four run periods of the experiment. Confidence intervals for the oscillation parameters $\Delta m_{32}^{2}, \sin ^{2} \theta_{23}, \sin ^{2} \theta_{13}$ and $\delta_{C P}$ are given for both normal and inverted hierarchy hypotheses. The combined result shows a slight preference for normal hierarchy. The second part presents the most recent results of the neutrino tau appearance analysis. The no-tau appearance hypothesis is excluded at $4.6 \sigma$ level, and the fluxaveraged inclusive charged-current $v_{\tau}$ cross-section is measured to be $(0.94 \pm 0.20) \times 10^{-38} \mathrm{~cm}^{2}$.

The 19th International Workshop on Neutrinos from Accelerators-NUFACT2017

25-30 September, 2017

Uppsala University, Uppsala, Sweden

\footnotetext{
* Speaker.

${ }^{\dagger}$ on behalf of the Super-Kamiokande collaboration
} 


\section{Introduction}

When cosmic rays interact with the Earth's atmosphere, they produce secondary and tertiary particles such as pions, kaons, and muons, which then decay into neutrinos. Atmospheric neutrinos are therefore a mix of $v_{e}, v_{\mu}, \bar{v}_{e}$, and $\bar{v}_{\mu}$ at production. Their fluxes are well known since they have been measured by several experiments such as shown in Fig.1. Because of the wide energy range covered by atmospheric neutrinos, it is possible to use them to study some of the remaining questions about neutrino physics, such as the mass hierarchy, $\theta_{23}$ octant and the CP-violating phase $\delta$.
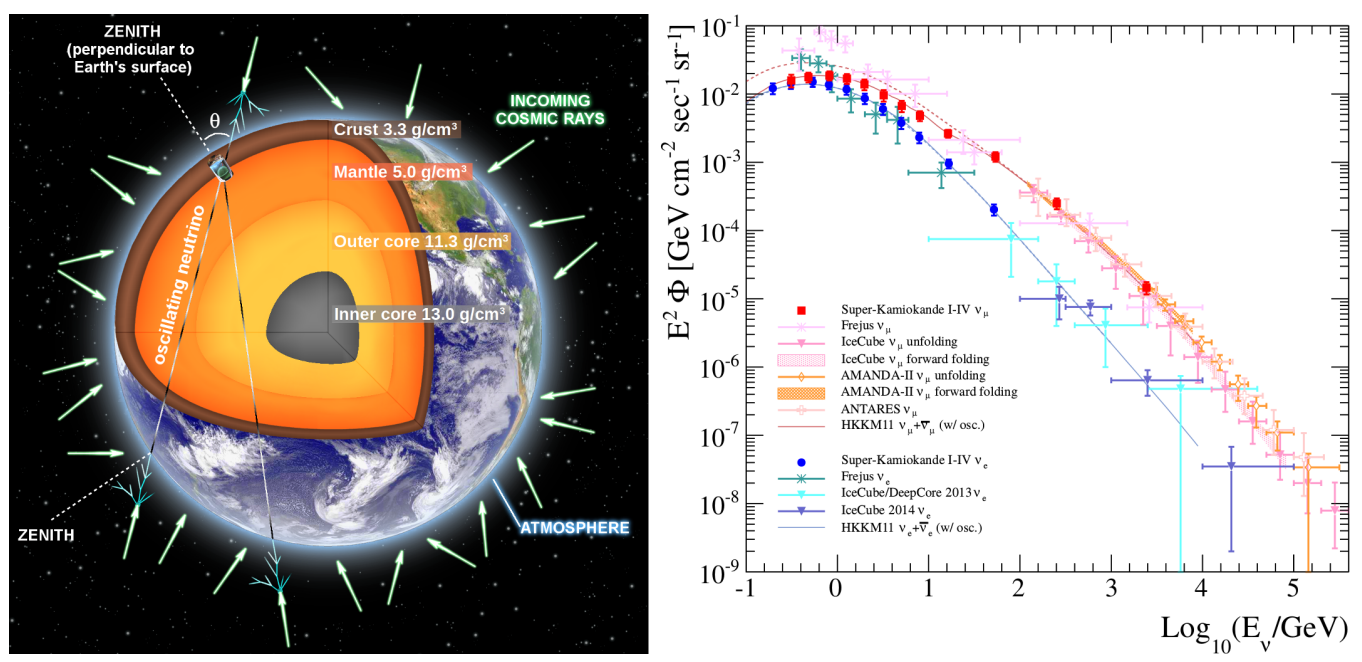

Figure 1: Left: Propagation of two neutrinos through the simplified Earth model used in the following analyses. Right: Atmospheric neutrino flux measured by Super-K (red) compared to other experiments. [1].

\section{The Super-Kamiokande experiment}

The Super-Kamiokande experiment is a 50-kton pure water Čerenkov detector located inside the Kamioka mine in the Gifu prefecture, Japan. It is divided into two distinct volumes. The first one, corresponding to the inner cylinder and called inner detector (ID), is instrumented with 11,126 20-inch Hamamatsu photomultiplier tubes (PMTs) and comprises a 22.5 kton fiducial volume. The second, called the outer detector (OD) and defined by the $2 \mathrm{~m}$ outer shell surrounding the ID, is instrumented with 1,885 8-inch Hamamatsu PMTs and is lined with Tyvek sheets to maximize light collection.

The experiment has been taking data since April 1996, with an average PMT coverage of $40 \%$. The results presented in the following sections include data from all four Super-K periods, presenting a total livetime of 5326 days.

In Super-Kamiokande, the atmospheric neutrino data samples are divided into three main types (Fig.2): 
- Fully Contained (FC) events, which have a reconstructed vertex inside the fiducial volume and no OD activity. Further sub-divided as a function of particle type (electron, muon or $\pi^{0}$-like), number of rings, energy, and number of decay electrons;

- Partially Contained (PC) events, which have a reconstructed vertex inside the fiducial volume but with OD activity. Sub-divided into stopping (in the OD) or through-going;

- Upward-going muon (Upmu) events.These are muons produced by neutrinos coming from the other side of the Earth which interact with the rock surrounding the detector. Sub-divided into stopping (in the ID), through-going non-showering, or through-going showering.
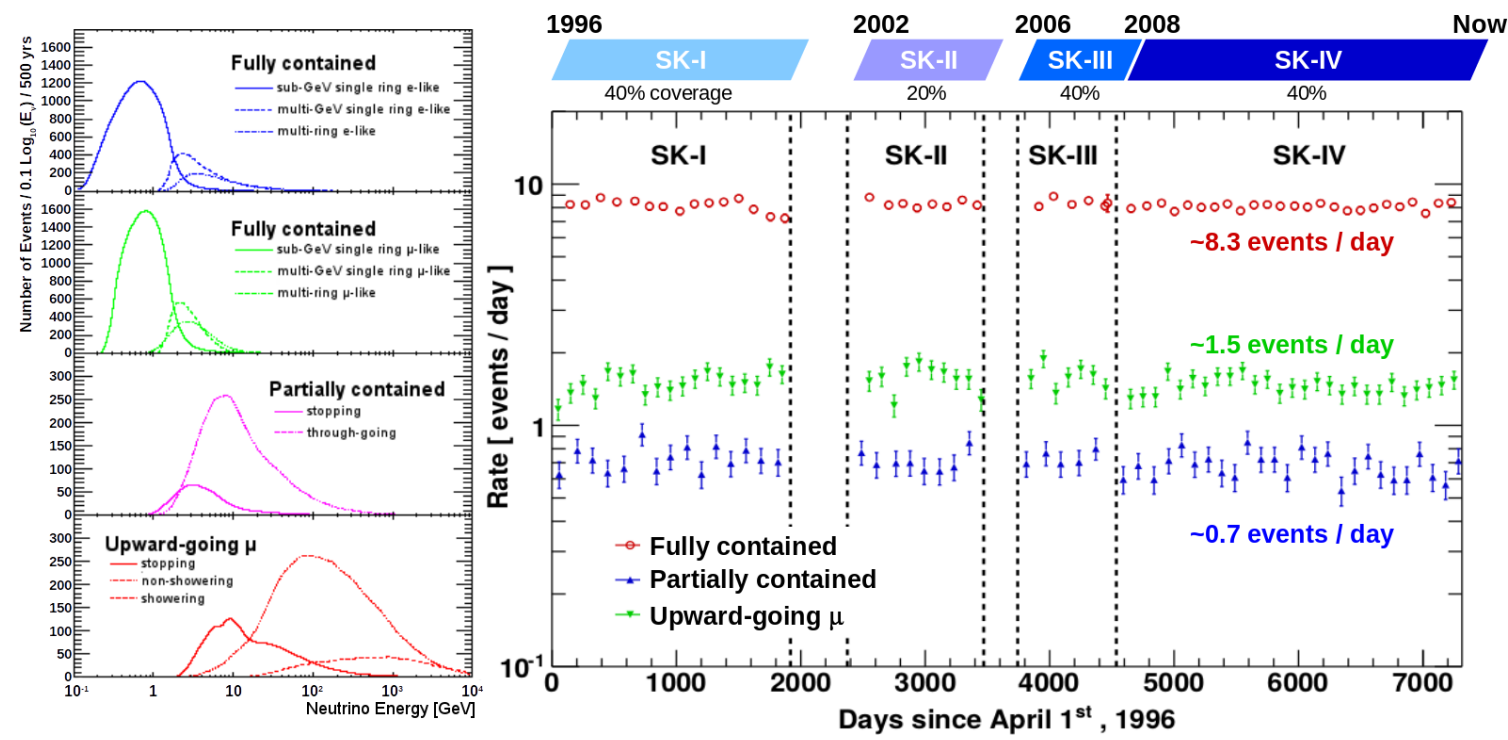

Figure 2: Left: Neutrino energy spectra per event sub-category. Right: Super-Kamiokande timeline (including PMT coverage) and daily rates per event type since beginning of data taking.

\section{Atmospheric neutrino oscillation analysis}

When crossing the Earth, $v_{e}$ scatter on electrons. It is possible to study the neutrino mass hierarchy by looking at how matter effects change the neutrino oscillation probabilities. When the mass hierarchy is normal, a resonant enhancement of $v_{\mu}$ disappearance / $v_{e}$ appearance in the few $\mathrm{GeV}$ region for upward-going neutrinos is expected. Vice-versa, if the mass hierarchy were inverted, such excess would be expected for anti-neutrinos instead. Experimentally this corresponds to observing an excess in the number of electrons appearing in Super-Kamiokande's few-GeV electron-like samples. A simplified version of the preliminary reference Earth model (PREM)[2] is used to take into account the electron density variations a neutrino traveling through the Earth can cross.

In the following studies, the solar neutrino oscillation parameters $\theta_{12}$ and $\Delta m_{21}^{2}$ are fixed. All fits are based on a binned chi-squared method where the systematic errors are propagated as Monte Carlo scaling factors on the expected number of events. The analyses include a total of 520 bins, 

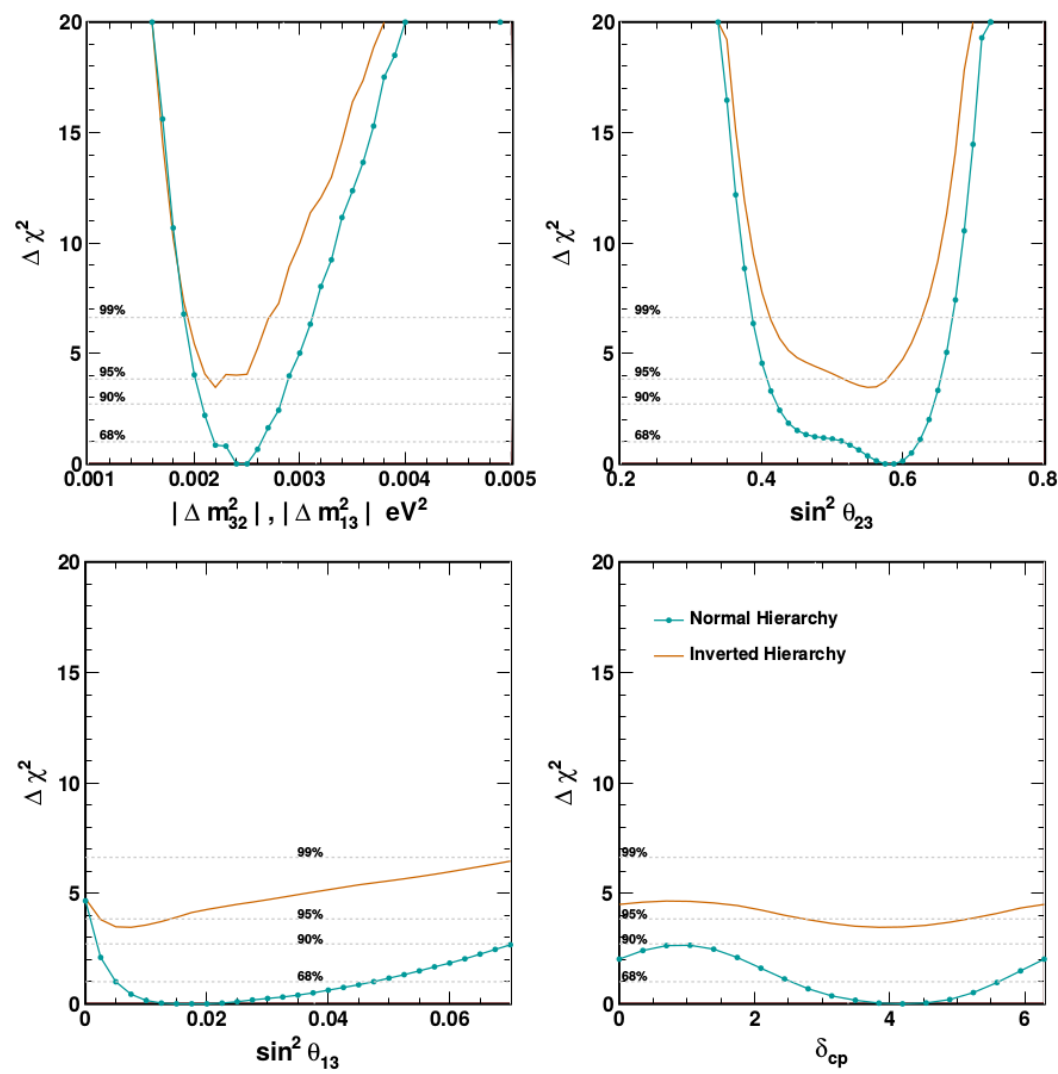

Figure 3: Normal and inverted hierarchy constraints on each of the atmospheric oscillation parameters when $\theta_{13}$ is not fixed. The inverted hierarchy results have been offset by the difference in the minimum $\chi^{2}$ values.

all four Super-K periods, and 155 systematic errors [3]. All data samples described in the previous section are used, with multi-GeV and multi-ring $v_{e}$ and $\bar{v}_{e}$ sub-samples being most sensitive to the mass hierarchy. Three different fits are done: without constraining $\theta_{13}$ (Fig.3), with $\sin ^{2} \theta_{13}$ fixed to the reactor neutrino experiment combined result 0.0219 \pm 0.0012 [4] (Fig.4), and finally with the additional T2K constraint (Fig.5). Best fits values for both mass hierarchy scenarios can be found in table 1 . In all cases, normal hierarchy is favored over inverted, as well as the second $\theta_{23}$ octant. The mass hierarchy preference gets stronger as more constraints are included. An additional fit is done for the second case, where an electron density scaling parameter $\alpha_{e}$ is added, $\alpha_{e}=0$ meaning neutrinos oscillate in vacuum and $\alpha_{e}=1$ for normal standard matter density. Results exclude vacuum oscillations at $1.6 \sigma$ level. In the third fit, the T2K constraint is done by reweighting the Super-Kamiokande atmospheric neutrino Monte-Carlo to the published T2K beam flux [5].

To interpret the significance of these results, the $C L_{s}$ method [6] is used. The minimal and maximal $C L_{s}$ values are obtained when the oscillation parameters are allowed to vary within their 90\% C.L.. With this approach, the inverted mass hierarchy is disfavored in the $\theta_{13}$ fixed cases by between $80.6 \%$ and $96.7 \%$ for the SK-only results, and by between $91.5 \%$ and $94.5 \%$ when the $\mathrm{T} 2 \mathrm{~K}$ constraint is added. 

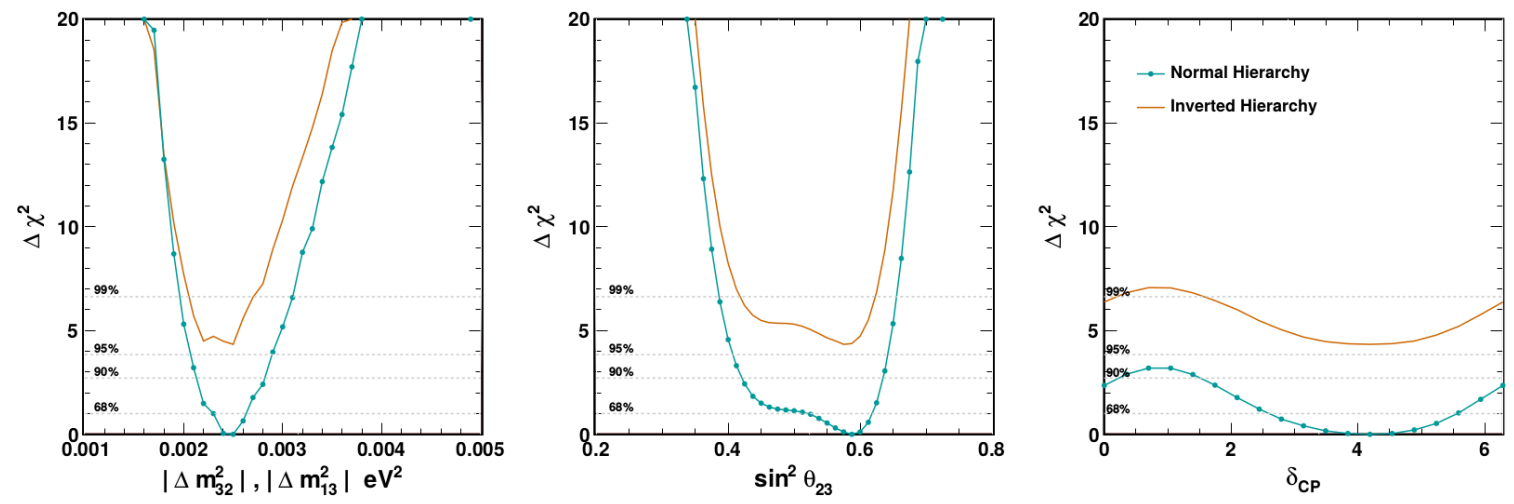

Figure 4: Constraints when $\theta_{13}$ is fixed to the reactor experiments result.
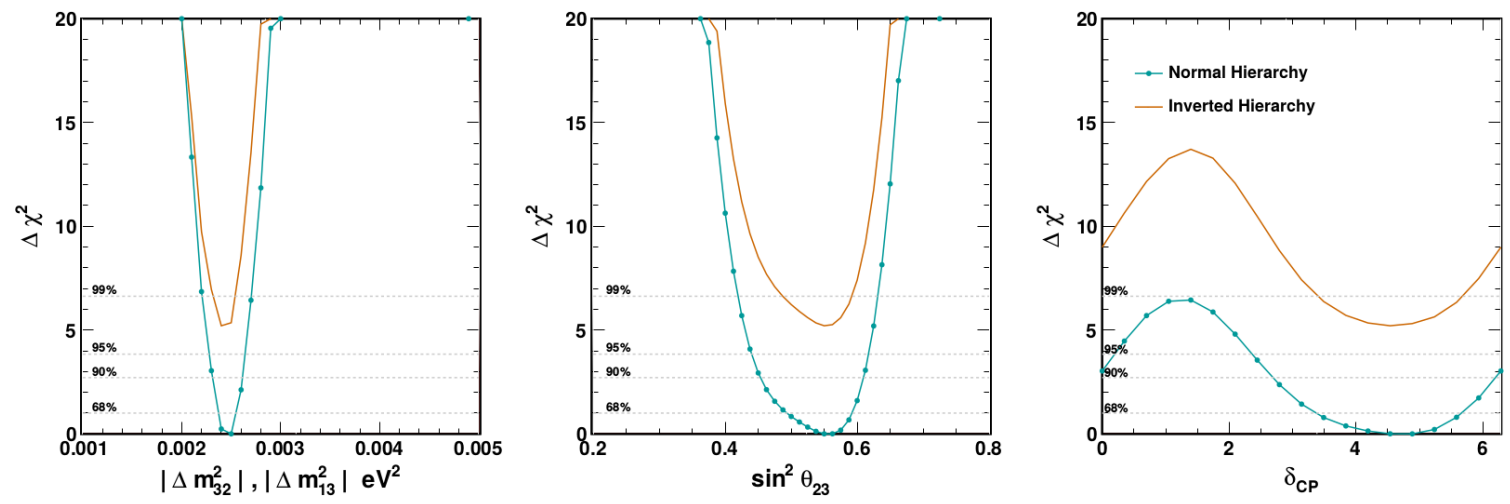

Figure 5: Constraints when $\theta_{13}$ is fixed and the T2K model is added.

\begin{tabular}{|c|c|c|c|c|c|c|}
\hline Fit & Hierarchy & $\chi^{2}$ & $\sin ^{2} \theta_{13}$ & $\sin ^{2} \theta_{23}$ & $\left|\Delta m_{32}^{2}\right|\left[\times 10^{-3} \mathrm{eV}^{2}\right]$ & $\delta_{C P}$ \\
\hline \multirow[t]{2}{*}{ SK $\theta_{13}$ Free } & $\mathrm{NH}$ & 571.7 & $0.019 \pm_{-0.014}^{+0.028}$ & $0.584 \pm_{-0.069}^{+0.039}$ & $2.47 \pm_{-0.28}^{+0.17}$ & $4.18 \pm_{-1.64}^{+1.43}$ \\
\hline & $\mathrm{IH}$ & 575.2 & $0.008 \pm_{-0.007}^{+0.017}$ & $0.550 \pm_{-0.078}^{+0.043}$ & $2.20 \pm_{-0.13}^{+0.33}$ & $3.85 \pm_{-2.15}^{+2.35}$ \\
\hline \multirow[t]{2}{*}{ SK $\theta_{13}$ Constrained } & $\mathrm{NH}$ & 571.7 & - & $0.588 \pm_{-0.067}^{+0.031}$ & $2.50 \pm_{-0.20}^{+0.13}$ & $4.19 \pm_{-1.59}^{+1.37}$ \\
\hline & $\mathrm{IH}$ & 576.1 & - & $0.575 \pm_{-0.085}^{+0.035}$ & $2.50 \pm_{-0.37}^{+0.08}$ & $4.19 \pm_{-1.63}^{+1.49}$ \\
\hline \multirow{2}{*}{$\mathrm{SK}+\mathrm{T} 2 \mathrm{~K} \theta_{13}$ Constrained } & $\mathrm{NH}$ & 639.9 & - & $0.550 \pm_{-0.059}^{+0.040}$ & $2.50 \pm_{-0.13}^{+0.05}$ & $4.89 \pm_{-1.45}^{+0.84}$ \\
\hline & $\mathrm{IH}$ & 644.8 & - & $0.550 \pm_{-0.049}^{+0.037}$ & $2.40 \pm_{-0.06}^{+0.13}$ & $4.54 \pm_{-0.96}^{+0.99}$ \\
\hline
\end{tabular}

Table 1: Best fit values and $\chi^{2}$ for all three analyses considering both mass hierarchy hypotheses.

\section{Tau neutrino appearance}

To study $v_{\tau}$ appearance [7], Super-Kamiokande searches for $v_{\tau}$ charged current interactions. Tau leptons have a short lifetime thus can only be indirectly detected. Most tau-decay final states have several outgoing particles, which are reconstructed as multi-ring events. The analysis focuses 
on selecting these multi-prong decays, specifically the fully-contained ones which have a visible energy larger than $1.3 \mathrm{GeV}$. This cut helps removing a large fraction of the low energy atmospheric background. Since it is difficult to tell apart a $v_{\tau}$ multi-ring event from background multi-ring events with standard cuts, a neural network based on seven discriminating variables is applied. The variables are: the visible energy since there is an energy threshold for tau production, particle identification of the most energetic ring, the number of decay electrons, the maximum distance between the primary vertex and any decay electron, the event's sphericity[8] since tau decays are relatively isotropic, the number of rings or ring fragment candidates, and finally the fraction of photoelectrons carried by the most energetic ring.
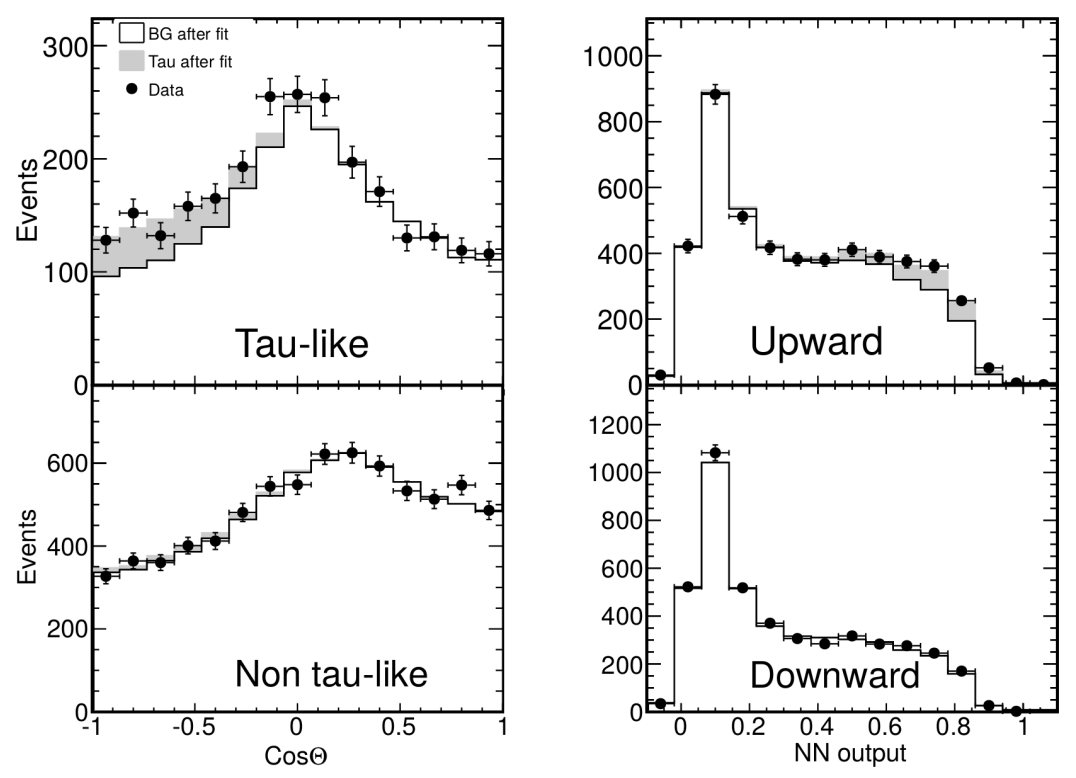

Figure 6: Fit results assuming normal hierarchy. The fitted tau signal is shown in gray.

The output of the neural network shows that there is a good separation between the tau signal and the background. Because $v_{\tau}$ appearance is only expected in the upward-going sample, downward-going data is used to validate the background simulation. We build PDFs using both the output of the neural network and the direction of the event, and use them to determine the tau normalization factor $\alpha_{\text {norm }}$ as described in Eq. 4.1, where $\varepsilon_{i}$ are the systematic error coefficients. The systematic uncertainties are taken from the 3-flavor oscillation analysis described in the previous section. Only the ones that change the signal and background histograms by at least $2.5 \%$ when applying a $1-\sigma$ variation are kept, leading to a total number of 28 systematic errors.

$$
N_{\text {data }}=\alpha_{\text {norm }} \times \text { Signal }+ \text { Background }+\sum_{i} \varepsilon_{i} \times\left(\text { Signal }_{i}+\text { Background }_{i}\right)
$$

The two-dimensional unbinned maximum likelihood fit returns $\alpha_{\text {norm }}=1.47 \pm 0.32$ (stat. + syst.) in the normal hierarchy scenario, which is equivalent to $338.1 \pm 72.7$ fully contained charged-current $v_{\tau}$ events. The no-tau appearance hypothesis is rejected at $4.6 \sigma$ level, which is better than the $3.3 \sigma$ initially expected. These results are mainly limited by statistics, given that a fit which does not include the systematic errors yields $\alpha_{\text {norm }}=1.41 \pm 0.28$. 
This result can also be interpreted in terms of cross-section, by multiplying the theoretical cross-section used in the Monte-Carlo by the normalization factor $\alpha_{\text {norm }}$. The flux-averaged theoretical cross-section between $3.5 \mathrm{GeV}$ and $70 \mathrm{GeV}$ predicted by NEUT[9] is $0.64 \times 10^{-38} \mathrm{~cm}^{2}$ thus the measured flux-averaged charged-current $v_{\tau}$ cross-section is $(0.94 \pm 0.20) \times 10^{-38} \mathrm{~cm}^{2}$ over the same energy range.

\section{Conclusion}

Super-Kamiokande has shown that mass hierarchy studies and a non-zero $\theta_{13}$ measurement could be done with atmospheric neutrinos only. In particular, the analyses show a preference for normal hierarchy over inverted as well as $\theta_{23}$ in the second octant. In the tau analysis, the no-tau appearance was excluded at $4.6 \sigma$ level in the normal hierarchy scenario.

\section{References}

[1] E. Richard et al. (Super-Kamiokande), Phys. Rev. D 94, 052001 (2016).

[2] A. M. Dziewonski and D. L. Anderson, Physics of the Earth and Planetary Interiors 25, 297 (1981).

[3] R. Wendell et al. (Super-Kamiokande), arXiv:1710.09126 [hep-ex].

[4] K. A. Olive et al. (Particle Data Group), Chin. Phys. C38, 090001 (2014).

[5] K. Abe et al. (T2K), Phys. Rev. D91, 072010 (2015), arXiv:1502.01550 [hep-ex].

[6] A. L. Read, Proceedings, Advanced Statistical Techniques in Particle Physics: Durham, UK, March 18-22, 2002, J. Phys. G28, 2693 (2002), [11(2002)].

[7] Z. Li et al. (Super-Kamiokande), arXiv:1711.09436 [hep-ex].

[8] T. Sjostrand, S. Mrenna, and P. Z. Skands, JHEP 05, 026 (2006), arXiv:hep-ph/0603175 [hep-ph].

[9] Y. Hayato, Proceedings, 1st International Workshop on Neutrino-nucleus interactions in the few GeV region (NuInt 01): Tsukuba, Japan, December 13-16, 2001, Nucl. Phys. Proc. Suppl. 112, 171 (2002), [171(2002)]. 